\title{
Investigation of adropin and IMA levels in psoriasis and their relation to duration and severity of disease
}

\section{Suzan Demir Pektas', Gokhan Pektas², Aynure Oztekin ${ }^{3}$, Tuba Gokdogan Edgunlu4, Sevim Karakas-Celik ${ }^{5}$, Salim Neselioglu ${ }^{6}$, Ozcan Erel ${ }^{6}$}

\begin{abstract}
${ }^{1}$ Department of Dermatology, Mugla Sitki Kocman University Faculty of Medicine, Mugla, Turkey, ${ }^{2}$ Department of Internal Medicine, Mugla Sitki Kocman University Faculty of Medicine, Trainig and Research Hospital, Mugla, Turkey, ${ }^{3}$ Department of Dermatology, Hitit University Faculty of Medicine, Corum Trainig and Research Hospital, Corum, Turkey, ${ }^{4}$ Department of Medical Biology, Mugla Sitki Kocman University Faculty of Medicine, Mugla, Turkey, ${ }^{5}$ Department of Molecular Biology and Genetic, Bulent Ecevit University Faculty of Science Zonguldak, Turkey, ${ }^{6}$ Department of Biochemistry, Yildirim Beyazit University, Faculty of Medicine Ankara, Turkey
\end{abstract}

Corresponding author: Dr. Suzan Demir Pektas, E-mail: suzandpektas@gmail.com

\begin{abstract}
Background: Psoriasis is an immunologically mediated and inflammatory skin disease, which is closely associated with some comorbid conditions such as cardiovascular disease and insuline resistance. It has identified that adropin is important for regulation of glucose, lipid metabolisms, energy and homeostasis. Ischemia Modified Albumin (IMA) generated by reactive oxidant radical is found to be sensitive marker of ischemic heart disease related to oxidative stress. We aimed adropin and IMA levels in psoriasis patients in comparison with healthy controls and their possible relation with duration and severity of disease. Materials and Methods: A total of 44 patients and 43 controls were included in this cross-sectional study, and disease severity was evaluated according to psoriasis area severity index (PASI) scoring. Demographic data, clinical features, anthropometric measures and laboratory findings were recorded in all study subjects. Serum adropin and IMA levels were measeured using enzyme-linked immunosorbent assay (ELISA) kit. Results: Psoriasis patients had higher values for IMA and C-reactive protein (CRP) compared to the control group. Adropin levels was decreased in the serum of psoriasis patients. The PV patients with PASI $>10$ had significantly lower adropin than psoriasis patients with PASI $\leq 10$, but had no significant different IMA levels between psoriasis patients with PASI $\leq 10$ and PASI $>10$. Duration and severity of disease and CRP levels positively correlated with IMA and negatively correlated with adropin in psoriasis patients.Conclusion: These findings indicate the relationship between psoriasis and significantly decreased adropin and increased IMA, along with chronic inflammation and oxidative stress, as associated mainly with long disease duration and severe disease.
\end{abstract}

Key words: Psoriasis; Adropin; Ischemia Modified Albumin

\section{INTRODUCTION}

Psoriasis is immune-mediated chronic and recurrent, sistemic inflammatory skin disease, which is closely associated with oxidative stress (OS). Most characteristic skin lesions are red, scaly sharply demarcated, indurated plaques $[1,2]$. The etiological and genetic factors for psoriasis is yet not exactly known. But some of reasons like skin infection, drugs, trauma, emotional stress, smoking and alcohol are influences the clinical development of psoriasis [1-3]. In the previous studies, psoriasis is reported to be related to systemic diseases such as hyperlipidemia, hypertension, insulin resistance (IR), metabolic and cardiovascular diseases (CVD) [4-10]. Psoriatic inflammation leads to development of these diseases $[5,10]$. In fact, psoriasis has been shown to be an independent risk factor for these systemic diseases $[4,10]$. The increased incidence of cardio-metabolic diseases in psoriasis is caused by underlying systemic inflammation together with

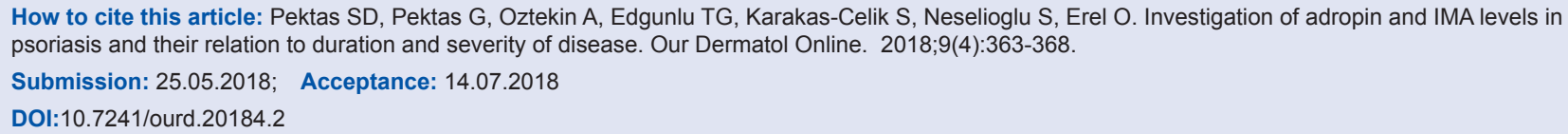


increased frequency of traditional cardio-metabolic diseases risk factors, which have been in patients with psoriasis $[4-7,10]$.

Adropin is identified protein encoded by the energy homeostasis-associated gene (En-ho) in the brain and liver [11]. It has a role in the maintenance of the insülin resistance and energy homeostasis, related to atherogenesis $[11,12]$. Adropin affects adiposity and is involved in preventing insülin resistance, dyslipidemia, and impaired glucose tolerance [12-14]. It has reported that increased level of adropin has been shown in various tissues of diabetic rats [14]. In another study, it has determined that lower adropin level leads to endothelial impairment and dysfunction [12]. Furthermore, low serum adropin introduced as a merker of clinically relevant coronary atherosclerosis [12-14].

Ischaemia-modified albumin (IMA), is measured by the albumin cobalt binding test, is reported as a promising marker for cardiac ischaemia $[15,16]$. Recent studies reported that IMA is also increased in cardio-metabolic diseases associated with OS such as hypercholesterolaemia, renal disease, polycystic ovary syndrome, obesity and type 2 diabetes mellitus [17-19]. It was reported that IMA levels are higher in patients with psoriasis than in healthy controls in patients with psoriasis in studies of Ozdemir M et al. [20], Isik S et al. [21] and Chandrashekar L et al. [22] Three studies reported that increased IMA levels of psoriasis patients is associated with increased systemic inflammation and OS in psoriasis [20-22].

We tried to to find outwhether serum adropin and IMA in patients with psoriasis, and to determine the relationship of serum adropin and IMA with demographic, clinical and laboratory characteristic.

\section{MATERIALS AND METHODS}

This cross-sectional study was performed in accordance with the guidelines of Helsinki Declaration and it was approved by the local ethical committee.

\section{Subjects}

This study reviews 44 patients with psoriasis who were admitted to the department of dermatology and 41 healthy volunteers. The patients with psoriasis plaques, the patients who received topical treatment for the last four weeks, the patients who received systemic treatment in the last three months, the patients with concurrent systemic disorders (coronary artery disease, liver failure, renal failure, malignancy etc), the patients who had a habit of smoking and/or alcohol consumption, the patients with pregnancy and the breastfeeding patients were excluded. Special care was exercised to match the study and control groups for age and sex. Demographic features, anthropometric measures and blood pressure values were recorded. Psoriasis duration was obtained by self report of the patients. Age, weight, height, and body mass index [BMI; weight (kilograms)/height (meters) $\left.{ }^{2}\right]$ were evaluated at baseline. fasting plasma glucose (FPG), CRP and lipid profile [total cholesterol, triglyceride, high density lipoprotein cholesterol (HDL-C), low density lipoprotein cholesterol (LDL-C)] at diagnosis as metabolic analysis were performed. Low density lipoprotein cholesterol (LDL-C) $(\mathrm{LDL}=$ total cholesterol- $[\mathrm{HDL}+($ Triglyceride/5)] $)$ were calculated as previously described. Disease severity was assessed by PASI and grouped as A PASI score below or equal 10 was defined as "mild disease" and above 10 was defined as "moderate-severe disease".

\section{Collection of Blood Samples}

Venous blood samples were collected in the early morning following a 8-hour-long fasting period. Sodium citrate and ethylenediamine tetraacetic acid (EDTA) were used as anticoagulants in the collection of these samples. It was made sure that none of the samples was icteric or hemolyzed. Then the samples were centrifuged at $3600 \mathrm{rpm}$ for 10 minutes and kept at $-80^{\circ} \mathrm{C}$ until analysis.

\section{Laboratory Analysis}

Serum CRP levels were measured by turbidimetry $(660 \mathrm{~nm} / 700 \mathrm{~nm})$ with a Cobas 6000 Analyzer (Roche Diagnostics, USA). Results were compared with those obtained using typical immunoturbidometry. FPG was measured with Cobas 6000 Analyzer (Roche Diagnostics, USA) by using the UV hexokinase method. Triglyceride and total cholesterol was determined with the enzymatic colorimetric assay, HDL-C was determined with the homogenous enzymatic colorimetric assay [23]. Serum IMA levels were measured by using colorimetric assay method previously described by Bar-Or et al. (24). This colorimetric method is based on biochemical properties of albumin to bind exogenous cobalt. In brief, $200 \mathrm{lL}$ of a subject serum was added to $50 \mathrm{lL}$ of $0.1 \%$ cobalt II chloride (CoCl2, 6H2O) (Sigma-Aldrich Chemie 
GmbH Riedstrasse 2, Steinheim, Germany) followed by mixing and 10 minutes of incubation in the dark at $37 \mathrm{C}$ to allow for cobalt albumin cobalt binding. Then, a total of $50 \mathrm{lL}$ dithiothreitol (DTT) were added as a coloring agent. After 2 minutes of incubation, $1 \mathrm{~mL}$ of 0.9 sodium chloride was added in order to reduce the binding capacity. The blank was prepared similarly with distilled water instead of DTT. The absorbance of samples was measured at $470 \mathrm{~nm}$ using a spectrophotometer (Jenway 6315 UV/visible Scanning Spectrophotometers, United Kingdom). IMA results were expressed in absorbance units (ABSUs). Each sample was measured in duplicate and the mean value was reported. Serum adropin levels were determined by ELISA method using an appropriate commercial kit (Cusabio Biotech Co., Wuhan, China). The minimum detectable dose of adropin was $0.0156 \mathrm{ng} / \mathrm{ml}$.

\section{Statistical Analysis}

To complete statistical assessments, the Statistical Package for Social Sciences (SPSS) software for Windows 18 (IBM SPSS Inc., Chicago, USA) was used. Continuous variables were expressed as mean \pm standard deviation. The normality of distribution of continuous variables was evaluated by the Kolmogorov-Smirnov, and therefore compared with independent sample Student t-test or MannWhitney U-test. Categorical variables were compared with chi-square statistic or Fisher's exact test when appropriate. The Pearson/Spearman correlation analysis was also used to analyze the relationship between numeric parameters. A p-value less than 0.05 was considered to be statistically significant.

\section{RESULTS}

The patient group included 44 psoriasis patients (20 males and 24 females). The control group included 41 age- and sex-matched healthy individuals ( 23 females and 18 males). The characteristics of the study groups are summarized in Table 1. Psoriasis patients had significantly higher values for BMI $\left(27.7 \pm 5.2 \mathrm{~kg} / \mathrm{m}^{2} \mathrm{vs}\right.$. $\left.23.3 \pm 1.4 \mathrm{~kg} / \mathrm{m}^{2}, \mathrm{p}<0.001\right)$ compared to the controls. Psoriasis patients had significantly higher mean FPG $(89 \pm 14.5 \mathrm{mg} / \mathrm{dL} v \mathrm{~s} .83 .8 \pm 8.2 \mathrm{mg} / \mathrm{dL}, \mathrm{p}<0.001$, Table 1$)$, CRP (19.9 $\pm 3.4 \mathrm{mg} / \mathrm{dL}$ vs. $3.4 \pm 0.8 \mathrm{mg} / \mathrm{dL}, \mathrm{p}<0.001$, Table 1) and triglyceride levels (126.6 $\pm 60.2 \mathrm{mg} / \mathrm{dL}$ vs. $104.2 \pm 55.5, \mathrm{p}<0.05$, Table 1) compared to the control group. When compared to controls, adropin levels (137.4 $\pm 49.2 \mathrm{ng} / \mathrm{ml}$ vs. $169.0 \pm 43.9 \mathrm{ng} / \mathrm{ml}, \mathrm{p}<0.001$, Table 1) were found to be lower in psoriasis patients
Table 1: Comparison in the characteristics of the control and psoriasis groups

\begin{tabular}{lccc}
\hline Parameters & $\begin{array}{c}\text { Control } \\
\text { group } \\
\text { N: } \mathbf{4 1}\end{array}$ & $\begin{array}{c}\text { Psoriasis } \\
\text { group } \\
\text { N: } \mathbf{4 4}\end{array}$ & $\boldsymbol{P}$ \\
\hline F/M & $23 / 18$ & $24 / 20$ & $\mathrm{NS}$ \\
Age (year) & $32.2 \pm 8.1$ & $33.5 \pm 8.7$ & $\mathrm{NS}$ \\
BMI (kg/m²) & $23.3 \pm 1.4$ & $27.7 \pm 5.2$ & $<0.001$ \\
Duration of disease (years) & - & $10.0 \pm 1.9$ & \\
PASI & - & $9.9 \pm 3.2$ & \\
CRP (mg/dL) & $3.4 \pm 0.8$ & $9.9 \pm 3.4$ & $<0.001$ \\
FPG (mg/dL) & $83.8 \pm 8.2$ & $89 \pm 14.5$ & $<0.001$ \\
LDL (mg/dL) & $94.2 \pm 34.7$ & $98.7 \pm 33$ & $\mathrm{NS}$ \\
Triglyceride (mg/dL) & $104.2 \pm 55.5$ & $126.6 \pm 60.2$ & $<0.05$ \\
TC (mg/dL) & $173.8 \pm 41.7$ & $175.5 \pm 38.1$ & $\mathrm{NS}$ \\
HDL (mg/dL) & $57.2 \pm 15$ & $51.9 \pm 15.3$ & $\mathrm{NS}$ \\
Ischaemia-modified & $0.4 \pm 0.1$ & $0.6 \pm 0.1$ & $<0.001$ \\
albümin (ABSU) & & & \\
Adropin (ng/ml) & $169.0 \pm 43.9$ & $137.4 \pm 49.2$ & $<0.001$ \\
\hline
\end{tabular}

Data werepresented as mean $\pm \mathrm{SD}$

$\mathrm{N}=$ number of volunteer; $\mathrm{NS}=$ =nonsignificant; $\mathrm{BMI}=$ body mass index; $\mathrm{F} / \mathrm{M}$ females/males; $\mathrm{PASI}=\mathrm{Psoriasis}$ area severity index; $\mathrm{FPG}=\mathrm{Fasting}$ plasma glucose; $C R P=C$-reactive protein; $L D L=$ low-density lipoprotein; $T C=T$ otal cholesterol; $\mathrm{HDL}=$ high density lipoprotein.

while IMA levels $(0.6 \pm 0.1 \mathrm{ABSU}$ vs. $0.4 \pm 0.1 \mathrm{ABSU}$, $\mathrm{p}<0.001$, Table 1$)$, were found to be higher in psoriasis patients.

According to disease severity, psoriasis patients were divided into two groups: 29 psoriasis patients with PASI $\leq 10$ (20 females and 7 males), 15 psoriasis patients with PASI $>10$ ( 6 females and 9 males). Table 2 summarizes the characteristics of the healthy controls, psoriasis patients with PASI $\leq 10$ and PASI $>10$. When compared to healthy controls, psoriasis patients with $\mathrm{PASI} \leq 10$ and PASI $>10$ had significantly higher BMI and FPG, $(\mathrm{p}<0.05$ for all, Table 2$)$. The psoriasis patients with $\mathrm{PASI} \leq 10$ and psoriasis patients with PASI $>10$ had significantly lower adropin values than healthy controls ( $<<0.05$ for all, Table 2$)$. The psoriasis patients with PASI $>10$ had significantly higher disease duration and PASI values than psoriasis patients with PASI $\leq 10(\mathrm{p}<0.001$ for both, Table 2$)$. The psoriasis patients with PASI $>10$ had significantly higher CRP values than psoriasis patients with PASI $\leq 10(\mathrm{p}<0.05$ for both, Table 2). The psoriasis patients with PASI $>10$ had significantly lower adropin levels than psoriasis patients with PASI $\leq 10(\mathrm{p}<0.05$, Table 2$)$. There were no statistically significant differences in IMA levels between the groups ( $\mathrm{p}>0.05$ for all, Table 2 ).

Table 3 shows the correlations among BMI, disease duration, PASI and CRP with adropin and IMA parameters in psoriasis patients. We founded that psoriasis patients had adropin was negatively correlated 
with duration of disease, PASI and CRP (r:-0.763, r: $-0,741$ and r:-0,682, $\mathrm{p}<0.001$ for all, Table 3). On the other hand, a positive correlation between IMA levels and duration of disease, PASI and CRP was observed (r:0.448, r:0.438 and r:0.439, p<0.05 for all, Table 3).

\section{DISCUSSION}

Psoriasis is a chronic and immune mediated skin disease accepted as a significant risk factor for CVD [5-7]. Adropin and IMA are accepted to partipicate a play role in the development and progression of some disease related to inflammation [11-13,15-19]. This study aimed to investigate how adropin and IMA levels are altered in psoriasis patients and whether adropin and IMA levels correlate with the severity and duration of psoriasis. In this study, patients with psoriasis were shown to have significantly lower levels of adropin and higher levels of IMA than healthy controls. We found a significant difference between the adropin levels of the patients with moderate to severe psoriasis and the patients with mild psoriasis, but there was no significant difference between the IMA levels of the two groups. It was founded that psoriasis patients had adropin levels was negatively correlated with disease duration and PASI, while IMA levels was positively correlation between disease duration and PASI.

Neutrophils seem to play an effective role to the development of inflammation and oxidative stres in this disease $[25,26]$. That is, inflammatory cytokines and OS markers are increased within tissues and peripheral circulation of the patients with psoriasis [26,27]. Inflammatory cytokines such as E-selectin, intracellular adhesion molecule-l, haptoglobin, interleukins (IL) and tumor necrosis factor-alpha (TNF- ) participate in the proliferation of keratinocytes within psoriatic lesions [25-27].

Adropin play a role in the maintenance of energy homeostasis and insulin response, closely related to atherogenesis [28]. Lower serum adropin level has recently been shown in studies investigating CVD risk leads to endothelial impairment and dysfunction, a marker for early event in atherogenesis and onset of CVD as well as a marker for clinically relevant coronary atherosclerosis [13]. It is well known that psoriatic inflammation leads to development of CVD and psoriasis has been an independent effector for CVD [5-9]. In this study, patients with psoriasis

Table 2: Comparation of the characteristics of the healthy controls, psoriasis patients with PASI $\leq 10$ and PASI>10

\begin{tabular}{|c|c|c|c|c|}
\hline Parameters & $\begin{array}{l}\text { Control } \\
(n=41)\end{array}$ & $\begin{array}{l}\text { Psoriasis patients with } \\
\text { PASI } \leq 10(n=29)\end{array}$ & $\begin{array}{l}\text { Psoriasis patients with } \\
\text { PASI>10 }(n=15)\end{array}$ & p-value \\
\hline F/M & $23 / 18$ & $20 / 7$ & $6 / 9$ & NS \\
\hline Age (year) & $32.29 \pm 8.1$ & $33.6 \pm 9.3$ & $33.1 \pm 7.8$ & NS \\
\hline $\mathrm{BMI}\left(\mathrm{kg} / \mathrm{m}^{2}\right)$ & $23.3 \pm 1.4$ & $28.1 \pm 5.2$ & $26.8 \pm 5.3$ & $<0.05 \dagger \ddagger$ \\
\hline Duration of disease (years) & & $9.4 \pm 1.8$ & $11.4 \pm 1.6$ & $0.001 \S$ \\
\hline PASI & & $8.1 \pm 1.2$ & $13.4 \pm 3.1$ & $<0.001 \S$ \\
\hline $\mathrm{CRP}(\mathrm{mg} / \mathrm{dL})$ & $3.4 \pm 0.8$ & $8.3 \pm 2.3$ & $13.1 \pm 3.0$ & $<0.05 \dagger \ddagger \S$ \\
\hline $\mathrm{FPG}(\mathrm{mg} / \mathrm{dL})$ & $83.8 \pm 8.2$ & $85.9 \pm 22.6$ & $90.6 \pm 7.7$ & $<0.05 \dagger \ddagger$ \\
\hline LDL (mg/dL) & $93.7 \pm 34.8$ & $99.2 \pm 29.7$ & $100.1 \pm 34.1$ & NS \\
\hline Triglyceride $(\mathrm{mg} / \mathrm{dL})$ & $104.2 \pm 55.5$ & $122.6 \pm 63$ & $128.7 \pm 59.7$ & NS \\
\hline $\mathrm{TC}(\mathrm{mg} / \mathrm{dL})$ & $173.8 \pm 41.7$ & $174.8 \pm 35.0$ & $177.8 \pm 44.7$ & NS \\
\hline $\mathrm{HDL}(\mathrm{mg} / \mathrm{dL})$ & $57.2 \pm 15$ & $55.4 \pm 17.1$ & $50.1 \pm 14.3$ & NS \\
\hline Ischaemia-modified albumin (ABSU) & $0.4 \pm 0.1$ & $0.6 \pm 0.0$ & $0.6 \pm 0.2$ & NS \\
\hline Adropin $(\mathrm{ng} / \mathrm{ml})$ & $169 \pm 43.9$ & $150.4 \pm 553.5$ & $112.2 \pm 26.4$ & $<0.05 \dagger \ddagger \S$ \\
\hline
\end{tabular}

Data werepresented as mean \pm SD

$\mathrm{N}=$ number of volunteer; $\mathrm{NS}=$ nonsignificant; $\mathrm{F} / \mathrm{M}=$ females/males; $\mathrm{PASI}=$ psoriasis area severity index; $\mathrm{BMI}=\mathrm{body}$ mass index; $\mathrm{FPG}=\mathrm{Fasting}$ plasma glucose; $\mathrm{CRP}=\mathrm{C}$-reactive protein; $\mathrm{LDL}=$ low density lipoprotein; $\mathrm{TC}=$ Total cholesterol; HDL=high density lipoprotein

Table 3: Correlation of adropin and ischaemia-modified albumin with characteristics in psoriasis patients

\begin{tabular}{|c|c|c|c|c|c|c|c|c|}
\hline & \multicolumn{2}{|c|}{ BMI } & \multicolumn{2}{|c|}{ Duration of disease } & \multicolumn{2}{|c|}{ PASI } & \multicolumn{2}{|c|}{ CRP } \\
\hline & $\mathbf{r}$ & $\mathbf{p}$ & $\mathbf{r}$ & $\mathbf{p}$ & $\mathbf{r}$ & $\mathbf{p}$ & $\mathbf{r}$ & $\mathbf{p}$ \\
\hline IMA & 0.167 & NS & $0.448^{\star *}$ & $<0.001$ & $0.438^{\star *}$ & $<0.001$ & $0.439^{\star \star}$ & ${ }^{\circ}<0.001$ \\
\hline Adropin & -0.177 & NS & $-0.763^{\star \star}$ & $<0.001$ & -0.741 & $<0.001$ & -0.682 & $<0.001$ \\
\hline
\end{tabular}

The $P$ values $<0.05$ were assessed as statistically significant; they were presented in bold.

IMA = ischaemia-modified albumin

$\mathrm{BMI}=$ body massindex; PASI = psoriasisareaseverityindex; CRP = c-reactive protein. 
who did not have any major CVD risk factors were shown to have higher levels of CRP and lower levels adropin than the controls lacking any majör CVD risk factors. Morever, we found that adropin levels of the patients with moderate to severe psoriasis lower than patients with mild psoriasis. The adropin correlated negatively with disease severity, disease duration and CRP in all psoriasis patients. The decresed adropin possibly reflects the association between psoriasis inflammation and CVD risk in psoriasis patients without any concomitant CVD risk factor presence.

The increase in the generation of reactive oxygen species (ROS) by neutrophils, keratinocytes and dermal fibroblasts plays a key factor in the pathogenesis of psoriasis. It has been postulated that the increase in the production of inflammatory cytokines by neutrophils, keratinocytes and dermal fibroblasts triggers oxidative stres [26,29-31].

IMA is an oxidatively modified form of albumin. IMA has been studied and regarded as a sensitive biomarker for the diagnosis of oxidatie stress (OS) related clinical conditions [16-19]. It was reported that IMA levels increase in the progression of metabolic syndrome [18], hyperlipidaemia [19], type 2 DM [32] myocardial infarction [33], coronary artery disease [34], which are also associated with psoriasis. Isik S et al. [21] reported that IMA increased in patients with psoriasis and it was correlated with disease duration but was not correlated with PASI. Ozdemi M et al. [20] reported that IMA levels are higher in patients with psoriasis than in healthy controls and IMA did not correlate with any disease characteristics. Chandrashekar L et al. [21] reported that serum IMA levels were significantly elevated and IMA showed a significant positive correlation with PASI score in psoriasis. In three study, reserchers reported that increased IMA levels support the role of OS role in the systemic inflammation seen in pathogenesis of co-morbidities associated with psoriasis, and especially with long disease duration and severe disease. Complying with studies of Isik S et al. [21], Ozdemir M et al. [20], Chandrashekar L et al. [21], we founded that psoriasis patients had significantly higher IMA levels than the controls. There were no statistically significant differences in IMA levels between the patients with moderate to severe and mild psoriasis. The IMA correlated positively with CRP as well as disease duration and PASI in all psoriasis patients. This findings suggested that increased IMA may reflect OS with associated chronic inflammation in patients with psoriasis and increased OS may contribute to development of CVD, as associated mainly with long term disease duration and severe disease. Such a contradictory finding of correlation according to other studies may be attributed to the differences in demographic and clinical characteristics of the reviewed patients, variations in biochemical measurement methods and heterogeneity in study populations.

In conclusion, we evaluated adropin and IMA levels and their relationship with duration and severity of disease in psoriasis patients in this study. We founded that IMA increased and adropin decreased in psoriasis patients. Cardiovascular risk factors such as higher BMI, dyslipidemia, FPG and CRP values were found to be more common in psoriasis patients. Additionally, psoriasis patients were demonstrated to have a significant positive correlation among IMA, disease duration, PASI and CRP paramaters, while a positive correlation among adropin, disease duration, PASI and CRP parameters. Chronic inflammation may play a role in the pathogenesis of increased OS in psoriasis. However, these findings should be interpreted carefully as the power of this study is limited by its relatively small cohort and lack of prospective data. In order to determine role of OS and sistemic inflammation on this disease or to understand the association between OS, psoriasis and metabolic disorders, further studies including large number of patients are needed.

\section{Statement of Human and Animal Rights}

All procedures followed were in accordance with the ethical standards of the responsible committee on human experimentation (institutional and national) and with the Helsinki Declaration of 1975, as revised in 2008 .

\section{Statement of Informed Consent}

Informed consent was obtained from all patients for being included in the study.

\section{REFERENCES}

1. Pujari VM, Ireddy S, Itagi I, Kumar H S. J Clin Diagn Res. The serum levels of malondialdehyde, vitamin e and erythrocyte catalase activity in psoriasispatients. 2014;8:CC14-6.

2. McGrath JA, Eady RA, Pope FM. Anatomy and organization of human skin. In: Burns T, Breathnach T, Cox N, Griffiths C (Eds). Rook's Textbook of Dermatology. Volume 1, ( $7^{\text {th }}$ ed.). Oxford United Kingdom: Blackwell Publishing; 2004; pp. 3.1- 3.84.

3. Khopkar U. Skin diseases and sexually transmitted infections. ( $5^{\text {th }}$ ed.). Mumbai: Bhalani Publisher (Book Depot); 2005. 
4. Dogan S, Atakan N. Red Blood Cell Distribution Width is a Reliable Marker of Inflammation in Plaque Psoriasis. Acta Dermatovenerol Croat. 017;25:26-31.

5. Menter A, Griffiths CE, Tebbey PW, Horn EJ, Sterry W. International Psoriasis Council. Exploring the association between cardiovascular and other disease related risk factors in the psoriasis population the need for increased understanding across the medical community. J Eur Acad Dermatol Venereol. 2010;24:1371-7.

6. Horreau C, Pouplard C, Brenaut E, Barnetche T, Misery L, Cribier B, et al. Cardiovascular morbidity and mortality in psoriasis and psoriatic arthritis: a systematic literature review. J Eur Acad Dermatol Venereol. 2013;27:12-29.

7. Prey S, Paul C, Bronsard V, Puzenat E, Gourraud PA, Aractingi S, et al. Cardiovascular risk factors in patients with plaque psoriasis: a systematic review of epidemiological studies. J Eur Acad Dermatol Venereol. 2010;24:23-30.

8. Hugh J, Van Voorhees AS, Nijhawan RI, Bagel J, Lebwohl M, Blauvelt A, et al. From the Medical Board of the National Psoriasis Foundation: The risk of cardiovascular disease in individuals with psoriasis and the potential impact of current therapies. J Am Acad Dermatol. 2014;70:168-77.

9. Miller IM, Skaaby T, Ellervik C, Jemec GB. Quantifying cardiovascular disease risk factors in patients with psoriasis: a metaanalysis. Br J Dermatol. 2013;169:1180-7.

10. Spah F. Inflammation in atherosclerosis and psoriasis: common pathogenic mechanisms and the potential for an integrated treatment approach. Br J Dermatol. 2008;159:10-7.

11. Bakry OA, El Hefnawy S, Mariee AH, El Gendy Y. Urinary Biopyrrins: A new marker of oxidative stress in psoriasis. Indian J Dermatol. 2016;61:169-73.

12. Lovren F, Pan Y, Quan A, Singh KK, Shukla PC, Gupta M, et al. Adropin is a novel regulator of endothelial function. Circulation. 2010;122(11 Suppl):S185-92.

13. Wu L, Fang J, Chen L, Zhao Z, Luo Y, Lin C, et al. Low serum adropin is associated with coronary atherosclerosis in type 2 diabetic and non-diabetic patients. Clin Chem Lab Med. 2014;52:751-8.

14. Aydin S, Kuloglu T, Aydin S, Eren MN, Yilmaz M, Kalayci M, et al. Expression of adropin in rat brain, cerebellum, kidneys, heart, liver, and pancreas in streptozotocin-İnduced diabetes. Mol Cell Biochem. 2013;380:73-81.

15. Bar-Or D, LauE, Winkler JV. Anovel assay for cobalt-albumin binding and its potential as a marker for myocardial ischemia a preliminary report. J Emerg Med. 2000;19:311-15.

16. Bhagavan NV, Lai EM, Rios PA, Yang J, Ortega-Lopez AM, Shinoda $\mathrm{H}$, et al. Evaluation of human serum albumin cobalt binding assay for the assessment of myocardial ischemia and myocardial infarction. Clin Chem. 2003;49:581-5

17. Piva SJ, Duarte MM, Da Cruz IB, Coelho AC, Moreira AP, Tonello R, et al. Ischemia-modifiedalbumin as an oxidative stress biomarker in obesity. Clin Biochem. 2011;44:345-47.

18. Caglar GS, Oztas E, Karadag D, Pabuccu R, Demirtas S. Ischemia-modified albumin and cardiovascular risk markers in polycystic ovary syndrome with or without insulin resistance. Fertil Steril. 2011;95:310-13.

19. Duarte MM, Rocha JB, Moresco RN, Bochi GV, Moresco RN, da Luz PL, et al. Association between ischemia-modified albumin, lipids and inflammation biomarkers in patients with hypercholesterolemia. Clin Biochem. 2009;42:666-71.

20. Ozdemir M, Kiyici A, Balevi A, Mevlitoğlu I, Peru C. Assessment of ischaemia-modified albumin level in patients with psoriasis._Clin
Exp Dermatol. 2012;37:610-4.

21. Işik S, Kılıç S, Öğretmen Z, Çakır DÜ, Türkön H, Cevizci S, Hız MM. The correlation between the psoriasis area severity index and ischemia-modified albumin, mean platelet volume levels in patients with psoriasis._Post Dermatol Alergol. 2016;33:290-3.

22. Chandrashekar L, Kumarit GR, Rajappa M, Revathy G, Munisamy M, Thappa DM. 25-hydroxy vitamin D and ischaemia-modified albumin levels in psoriasis and their association with disease severity. Br J Biomed Sci. 2015;72:56-60.

23. Friedewald WT, Levy RI, Fredrickson DS. Estimation of the concentration of low-density lipoprotein cholesterol in plasma, without use of the preparative ultracentrifuge. Clin Chem. 1972;18:499-502.

24. Turell L, Radi R, Alvarez B. The thiol pool in human plasma: the central contribution of albumin to redox processes. Free Radic Biol Med. 2013;65:244-53.

25. Emre S, Demirseren DD, Alisik M, Aktas A, Neselioglu S, Erel O. Dynamic thiol/disulfide homeostasis and effects of smoking on homeostasis parameters in patients with psoriasis. Cutan Ocul Toxicol. 2017;25:1-4.

26. Rocha-Pereira P, Santos-Silva A, Rebelo I, Figueiredo A, Quintanilha A, Teixeira F. The inflammatory response in mild and in severe psoriasis. Br J Dermatol. 2004;150:917-14.

27. Arican O, Aral M, Sasmaz S, Ciragil P. Serum levels of TNF-alpha, IFN gamma, IL-6, IL-8, IL-12, IL-17, and IL-18 in patients with active psoriasis and correlation with disease severity. Mediators Inflamm. 2005;2005:273-9.

28. Kumar KG, Trevaskis JL, Lam DD, Sutton GM, Koza RA, Chouljenko VN, et al. Identification of adropin as a secreted factor linking dietary macronutrient intake with energy homeostasis and lipid metabolism. Cell Metab. 2008;8:468-81.

29. Briganti S, Picardo M. Antioxidant activity, lipid peroxidation and skin diseases. What'snew. J Eur Acad Dermatol Venereol. 2003;17:663-9.

30. Young CN, Koepke JI, Terlecky LJ, Borkin MS, Boyd Savoy L, Terlecky SR. Reactive oxygen species in tumor necrosis factoralpha-activated primary human keratinocytes: implications for psoriasis and inflammatory skin disease. J Invest Dermatol. 2008;128:2606-14.

31. Pietrzak AT, Zalewska A, Chodorowska G, Krasowska D, MichalakStoma A, Nockowski P, et al. Cytokines and anticytokines in psoriasis. Clin Chim Acta. 2008;394:7-21.

32. Topuz M, Celik A, Aslantas T, Demir AK, Aydin S, Aydin S. Plasma adropin levels predict endothelial dysfunction like flow-mediated dilatation in patients with type 2 diabetes mellitus. J Invest Med. 2013;61:1161-4.

33. Aydin S, Kuloglu T, Aydin S, Kalayci M, Yilmaz M, Çakmak T, et al. Elevated adropin: a candidate diagnostic marker for myocardial infarction in conjunction with troponin-I. Peptides. 2014;58:91-7.

34. Zhang C, Zhao L, Xu W, Li J, Wang B, Gu X, et al. Correlation of serum adropin level with coronary artery disease. Zhonghua Yi Xue Za Zhi. 2014;94:1255-7.

Copyright by Suzan Demir Pektas, et al. This is an open-access article distributed under the terms of the Creative Commons Attribution License, which permits unrestricted use, distribution, and reproduction in any medium, provided the original author and source are credited. Source of Support: Nil, Conflict of Interest: None declared. 